\title{
LAS NOCIONES DE LENGUAJE IMPLÍCITAS EN LAS TEORÍAS LOCALIZACIONISTA Y GLOBAL: UN ACERCAMIENTO DESDE LOS ESTUDIOS DE LAS AFASIAS
}

\author{
Anita Arrieta Espinoza
}

\begin{abstract}
RESUMEN
El objetivo de este artículo es presentar textos relacionados con el estudio de la afasia para señalar en ellos elementos que permitan elaborar una concepción de lenguaje influenciada por las propuestas de las teorías localizacionista y global.

Palabras clave: afasia, lenguaje, teoría localizacionista, teoría global, psicolingüística.
\end{abstract}

\begin{abstract}
The purpose of this article is to present related texts that focus on aphasia in order to highlight elements within them that allow for the elaboration of a concept of language influenced by both locally and globally proposed theories.

Key words: aphasia, language, localized theories, global theories, psycholinguistics.
\end{abstract}

\section{Introducción}

En la etapa actual de los estudios psicolingüísticos, no existe una preocupación particular por definir el concepto de lenguaje. Se cuenta con un presupuesto medular que sostiene una vasta investigación: el lenguaje como actividad observable a partir del estudio del procesamiento mental.

En esta disciplina, cuyo objetivo es el estudio de los procesos mentales implicados en la comprensión y producción del lenguaje, tanto en sujetos "normales" como en sujetos "que han

\footnotetext{
Anita Arrieta Espinoza. Profesora de la Escuela de Filología, Linguística y Literatura de la Universidad de Costa Rica. San Pedro, San José, Costa Rica.

Correo electrónico: aarrieta@le.ucr.ac.cr, aarrietae@yahoo.com
}

Recepción: 17- 4- 2008

Aceptación: 11- 7- 2008 
perdido sus habilidades lingüísticas", cabría suponer que no existe polémica en lo relacionado con la manera de definir el término lenguaje.

No obstante, hemos percibido, en los textos relacionados con las afasias ${ }^{1}$, que el concepto de lenguaje a partir del cual se trabaja, aparece apenas esbozado.

Hemos observado también que, a pesar de lo no explícito del concepto de lenguaje manejado en los textos, éste parece estar influenciado por los lineamientos de las teorías localizacionista ${ }^{2}$ y global $^{3}$.

Con el fin de determinar en qué medida las observaciones anteriormente planteadas pueden ser confirmadas, nos interesa en este artículo acercarnos a los textos relacionados con la afasia.

Pretendemos, con base en la recopilación de textos relacionados con el tema, encontrar elementos que nos permitan delinear un concepto de lenguaje más próximo o menos próximo a las definiciones de lenguaje propuestas, de manera implícita y explícita, por las teorías localizacionista y global.

Hemos escogido estas teorías, llamadas "clásicas" en la historia de los estudios de corte neuropsicológico, dada su fuerte repercusión en la manera en que se ha definido y abordado el estudio de la afasia.

La influencia de estas líneas de pensamiento ha incidido en la escogencia de los presupuestos teóricos que operan en la elaboración de tests, diagnósticos y rehabilitaciones en el tratamiento de pacientes afásicos, sujetos claves en la investigación psicolingüística.

Para ubicar el trabajo psicolingüístico con las "patologías de lenguaje", dedicamos la primera parte de este texto a presentar los presupuestos (sobre el lenguaje, la dimensión comportamental y las fuentes de datos) con los que opera esta disciplina.

Luego, nos referimos a lo que hemos denominado "núcleos de las teorías localizacionista y global en lo referente al concepto de lenguaje".

Y finalmente, organizamos los textos escogidos dentro de los núcleos planteados, con el fin de analizar en qué medida estos textos nos permiten confirmar o desechar la influencia de concepciones de lenguaje de corte localizacionista, global o mixto.

\section{Algunos presupuestos de la psicolingüística}

\subsection{El concepto de lenguaje}

Antes de proceder a exponer y analizar los datos que podrían permitirnos un acercamiento a la noción de lenguaje propuesta por las teorías localizacionista y global, consideramos necesario exponer, en forma suscinta, los presupuestos sobre el lenguaje con los que trabaja la psicolingüística.

Por la precisión de los datos, y sobre todo por la naturaleza introductoria del texto, hemos escogido como punto de partida para esta sección la información suministrada por Belinchón ${ }^{4}$, M. et al. (1992).

Estos autores señalan la dificultad que existe para definir con exactitud el término lenguaje:

El término lenguaje es un término polisémico y ambiguo y los límites de su definición vulgar (i.e. no científica) son borrosos e imprecisos. Al mismo tiempo, la multiplicidad y variedad de sus usos sugiere la 
posibilidad de que el término lenguaje remita a una función que puede ser analizada desde muy diferentes perspectivas, en relación con muy diferentes tipos de organismos, y por referencia a dimensiones de análisis de muy diversa naturaleza ${ }^{5}$. lo siguiente:

Además, estos investigadores evalúan algunas definiciones de lenguaje y observan

Pese a su disparidad, estas definiciones de lenguaje permiten entrever también ciertas regularidades. Estas definiciones dan cuenta de algunos de los siguientes hechos: a) En primer lugar, el hecho de que el lenguaje pueda interpretarse como un sistema compuesto por unidades -los signos lingüísticos- cuya organización interna puede ser objeto de una descripción estructural o formal; b) en segundo lugar, el hecho de que la adquisición y uso de un lenguaje por parte de los organismos posibilita en éstos formas peculiares y específicas de relación y de acción sobre el medio (especialmente sobre el medio social), y el hecho de que el lenguaje se materializa en, y da lugar a, formas concretas de conducta, lo que permite interpretarlo, también, como una modalidad o tipo de comportamiento ${ }^{6}$.

Para concluir que:

El énfasis en una u otra (vertiente del fenómeno lingüístico), y también el distinto modo de interpretar su mutua dependencia o independencia lógica, permiten (...) observar las señas de identidad generales de las distintas disciplinas interesadas en el estudio del lenguaje y objetivar también algunos de los puntos de acuerdo o desacuerdo que existen entre las teorías elaboradas desde un mismo marco disciplinar ${ }^{7}$.

De acuerdo con el objetivo de este artículo, coincidimos con Belinchón et al. en la naturaleza ambigua y polisémica del concepto de lenguaje. Subrayamos también el hecho de que este concepto pueda materializarse en formas concretas de actividades linguísticas interpretables, aun dentro de un mismo marco disciplinario, con matices variados.

Es precisamente en esta línea donde se sitúa este artículo: en el intento de rastrear la manera en la que el término "lenguaje" se ha definido, en una misma disciplina, desde perspectivas localizacionistas, globales o, incluso, híbridas.

\subsection{La dimensión comportamental del lenguaje}

Esta dimensión ${ }^{8}$ implica considerar el lenguaje como un tipo de actividad o comportamiento que ejecuta "un sujeto".

Según esta interpretación del concepto de lenguaje, la noción de sujeto (agente que desempeña una actividad lingüística) es fundamental. Este sujeto domina un sistema de signos y símbolos, como consecuencia de un dispositivo genético particular y/o de un proceso de aprendizaje en la interacción con otros miembros de su especie.

A través de ese sistema, el sujeto puede completar y desarrollar un repertorio de acciones y respuestas sobre el medio.

El lenguaje es definido como una actividad que puede adoptar dos modalidades que se realizan de forma simultánea y combinada durante la conversación: la comprensión y la producción.

Estas modalidades se han descrito desde el plano neuroanatómico y fisiológico, mediante la identificación de las estructuras neuroanatómicas y fisiológicas que participan en la producción y comprensión de las señales linguiísticas. Se han descrito también desde un plano cognitivo, haciendo énfasis en el estudio de las representaciones y procesos internos (mentales) que subyacen a la comprensión y producción del lenguaje.

De esta manera, desde la perspectiva psicológica de la investigación, propia de la psicolingüística, existe el supuesto de que la actividad linguiística implica mecanismos y regularidades explicables desde las descripciones neurofisiológicas y desde las descripciones cognitivas. 
El interés radica en precisar el conjunto de operaciones mentales que un sujeto ejecuta para seleccionar los signos relevantes a su intención comunicativa. Para esto, delimita cómo se efectúan cada una de las etapas de los procesos de comprensión y de producción del lenguaje, así como cada una de las operaciones mentales involucradas en las actividades lingüísticas.

\title{
2.3. Fuentes de datos con las que trabaja la perspectiva comportamental del lenguaje en psicolingüística: los pacientes afásicos
}

Los objetivos $^{9}$ de la psicología del lenguaje (psicolingüística) se han organizado en tres grandes ejes: 1) El estudio de las actividades de producción y de comprensión del lenguaje; 2) el estudio de las funciones cognitivas que desempeña el lenguaje y que sirven de soporte a su uso y adquisición; 3) el estudio de los procesos de adquisición y deterioro de las funciones y modalidades de la actividad lingüística, es decir, el lenguaje en sus aspectos evolutivos y patológicos.

El eje número tres es el que nos preocupa en este artículo. Los datos extraídos de la investigación clínica con pacientes afásicos constituyen una vía para el conocimiento del lenguaje como sistema mental. Lo que se ha denominado "alteraciones del lenguaje normal", ha significado una fuente de datos importante para el conocimiento de los procesos y estructuras mentales que se emplean en la actividad lingüística; de ahí la escogencia, en este artículo, de los textos relacionados con la afasia y con las teorías localizacionista y global.

Al respecto, la citas de Belinchón et al. (1992: 701 y 702):

Dada la "invisibilidad fenomenológica" de la inmensa mayoría de los mecanismos cognitivos que intervienen en la actividad lingüística, las alteraciones del lenguaje revelan, con especial relieve, la existencia de esos mecanismos. La anomalía o, paradójicamente, la ausencia de partes de esa maquinaria desvelan, con frecuencia, su existencia subyacente (...) así también, los daños o ausencias de mecanismos que nos permiten comunicarnos simbólicamente, decir y comprender (o leer y comprender lo que leemos) hacen patente, con gran fuerza, tanto la existencia como la complejidad de tales mecanismos.

\begin{abstract}
Más aún: las deficiencias y trastornos del lenguaje pueden proporcionar también claves importantes en relación con la naturaleza de los procesos psicolingüísticos, la estructura de los procesos psicolingüísticos, la estructura del sistema mental responsable del lenguaje y las relaciones entre ese sistema y otros subsistemas de la mente. De este modo, las investigaciones sobre las alteraciones del lenguaje forman parte de la empresa psicolingüística como un todo, en tanto que ésta tiene precisamente el objetivo de definir el sistema mental subyacente al lenguaje y sus relaciones con otros sistemas, Así, por ejemplo, el hecho de que distintos componentes del lenguaje se dañen de forma selectiva en los trastornos afásicos parece apoyar una concepción de ese sistema mental como compuesto de subsistemas específicos (módulos de procesamiento), relativamente independientes entre sí, al menos en algunos casos y en buena parte de su funcionamiento. Y, en lo que se refiere a las relaciones entre el sistema lingüístico y otros subsistemas mentales, las investigaciones sobre autismo y psicosis, por ejemplo, revelan con especial relieve las estrechas interacciones y lazos de dependencia entre los aspectos pragmáticos de la producción y la comprensión lingüísticas, por una parte, y ciertos mecanismos que parecen estar especializados en el pensamiento interpersonal o la atribución e inferencia de estados mentales en los locutores, por otra.
\end{abstract}

Tal como se deduce de la teoría antes citada, existe un presupuesto base: el lenguaje es considerado como actividad mental y, a partir de esta definición, cada tendencia aclara, modifica y adecua este concepto según sus objetivos particulares.

En este artículo, consideramos que, además de lo ya expuesto, es importante señalar que no siempre las tendencias "aclaran, modifican y adecuan" de manera clara sus presupuestos, porque sería negar la posible (y además enriquecedora) confluencia de discursos diversos en las prácticas de investigación. 
En el caso de lo que nos preocupa en este artículo, hemos observado que, en muchos de los textos relacionados con la afasia, confluyen, de manera a veces armoniosa, a veces casual, tendencias catalogadas como teóricamente excluyentes.

Por ejemplo, algunas investigaciones presentan una línea teórica en la manera de revisar los antecedentes; pero, en la forma de analizar los resultados, se lee una posición híbrida, o incluso contradictoria a la propuesta teórica inicial, sin que se explique el por qué de tal procedimiento.

Mencionamos esta situación porque consideramos que es en esta coexistencia de discursos donde realmente se sitúa la polémica entre las propuestas de lenguaje provenientes de las teorías localizacionista y global, tema que nos interesa en este trabajo.

\section{Metodología}

En este artículo, nos proponemos reunir y analizar una muestra de textos que nos permitan rastrear, a partir de las alusiones al concepto de lenguaje, la influencia de los presupuestos localizacionistas y globales.

Debido a que en la mayoría de las fuentes consultadas no aparecen las definiciones de lenguaje de manera explícita, hemos intentado presentar los textos de modo que la información no pierda su naturaleza "de esbozo" ni de "no explícita", con el fin de analizar, en un futuro, esta situación.

Los textos escogidos son fragmentos de diferentes artículos científicos relacionados con la afasia, en los que se investigan aspectos relacionados con el lenguaje. También incluimos textos en los que percibimos una intención manifiesta de discutir sobre el concepto de lenguaje.

Para efectos de organización del trabajo, hemos clasificado los textos a partir de los grandes núcleos de las teorías localizacionista y global en lo referente al lenguaje. A partir de esos elementos, intentamos señalar, en el material de trabajo, la influencia de las teorías en estudio sobre las alusiones al concepto de lenguaje.

La escogencia de los textos en lo relativo a tipo de texto y cantidad de la muestra ha sido motivada por el grado en el que aporten material para los objetivos de esta investigación.

\section{Definiciones de lenguaje relacionadas con la teoría localizacionista}

Al inicio de esta investigación, pretendíamos encontrar definiciones específicas de lenguaje, propuestas por la teoría localizacionista. Esperábamos, a partir de ese dato, analizar si los textos estudiados iban en la misma línea que la definición propuesta por esa teoría.

Sin embargo, no encontramos en los textos consultados ninguna definición específica de lenguaje postulada por la teoría localizacionista.

En su lugar, hallamos numerosas alusiones al término lenguaje, tales como "el lenguaje está depositado en el cerebro", "el hemisferio izquierdo es donde están localizadas las áreas relacionadas con el lenguaje" y otras del mismo tipo.

Ante esta situación, consideramos que lo señalado al final del apartado 2.3. de este texto, se hizo todavía más notorio: la teoría localizacionista se menciona como una teoría clásica en los estudios de corte neuropsicológico; no obstante, sus presupuestos, en lo relativo al lenguaje, no aparecen "claramente esbozados".

Esa situación nos permitió, en términos de los objetivos de este artículo, confirmar en cierto grado las observaciones de las cuales parte esta investigación. Y, en términos de 
procedimientos, implicó la búsqueda de algunos ejes que nos permitieran organizar y analizar la información consultada, con el fin de acercarnos a una definición de lenguaje en la línea de la teoría localizacionista.

Para ello, después de una revisión teórica, escogimos los siguientes "núcleos"10 como ordenadores de los textos en estudio:

a) El lenguaje está depositado materialmente en el cerebro, en ciertas zonas de la masa cerebral. Solo algunas áreas del cerebro están implicadas en la actividad lingüística, como el área de Broca.

b) El procesamiento del lenguaje se ubica en uno de los dos hemisferios, específicamente en el izquierdo.

c) Existe una conexión paralela entre cada tarea linguiística y cambios químicos, celulares y configuracionales en áreas cerebrales particulares. Se pueden aislar las funciones psicolingüísticas.

Procedemos, en el apartado siguiente, a presentar textos relacionados con la investigación sobre afasia. Hemos agrupado cada uno de los textos escogidos dentro de alguno de "los grandes núcleos" de la teoría localizacionista, con el fin de destacar los elementos que se acerquen a los planteamientos de la teoría mencionada.

\subsection{Definiciones relacionadas con el núcleo 1}

En relación con el primer núcleo: 'El lenguaje está depositado materialmente en el cerebro, en ciertas zonas de la masa cerebral. Solo algunas áreas del cerebro están implicadas en la actividad lingüística, como el área de Broca', hemos reunido los siguientes textos:

\footnotetext{
La teoría (se refiere a la localizacionista) afirma, en su forma más extrema, que existe una relación unoa uno entre las áreas anatómicas del cerebro y su función en el cuerpo. En este sentido, se deriva de la posición de Broca ${ }^{11}$, la primera formulada en 1861 con referencia al habla, y más tarde extendida a otras funciones (Crystal 1993: 121).
}

En el texto anterior se mencionan los términos 'Broca', 'habla' y 'otras funciones'. Estas tres palabras son las que podemos señalar como acercamientos a una posible concepción del término 'lenguaje'.

En un primer momento, pareciera que, por la mención de Broca y de su reflexión sobre el 'habla', se estaría diciendo que el concepto de lenguaje con el que trabaja la teoría localizacionista equivaldría a 'habla'.

Sin embargo, si contextualizamos la cita anterior dentro de otras con información histórica, observamos que, cuando se iniciaron las investigaciones localizacionistas, la definición de lenguaje no era equivalente a habla.

Más bien, la definición de lenguaje era un punto importante de discusión. Esta definición debía ser aclarada. Por lo novedoso de los datos encontrados en la época, dentro del marco de una disciplina particular, no se encontraba una definición de lenguaje que reuniera elementos relacionados con las manifestaciones (o ausencia de manifestaciones) linguiísticas de los pacientes estudiados por los investigadores.

Si completamos la cita anterior con la de Messerli (1989: 26), la situación se presenta de una manera más clara:

En agosto del mismo año (1861), el caso Leborgne se presentó de manera más circunstancial, en la

Sociedad de Anatomía esta vez. El título es modesto: 'Anotaciones sobre el lugar de la facultad del 
lenguaje articulado, seguido de una observación de afemia', pero el interés de esta comunicación reside en el hecho de que, por primera vez, Broca se expresa sobre su concepción de lenguaje. Para él, existen varias especies de lenguaje pero todo sistema de signos que permita expresar ideas es un lenguaje en el sentido general del término; ' Hay una facultad general del lenguaje que preside todos las maneras de expresar pensamiento, y que puede ser definida: la facultad de establecer una relación constante entre una idea y un signo, ya sea un sonido, un gesto, una figura o un trazo cualquiera (331). Existen casos en los cuales la facultad general de lenguaje persiste inalterada (...) y en los cuales, por lo tanto, una lesión cerebral elimina el lenguaje articulado. Esta abolición del habla en los individuos que no tienen parálisis ni son idiotas, constituye un síntoma bastante singular para el cual parece útil designarlo con un nombre especial. Yo le daría pues el nombre de afemia; pues lo que les falta a estos enfermos, es solamente la facultad de articular las palabras. Ellos entienden todo lo que se les dice (332).

Dentro de esta misma línea, ubicamos el siguiente texto. En él, de una manera específica, se discute sobre una definición de lenguaje (novedosa en los estudios pioneros sobre la localización de la afasia en el cerebro).

Esta definición es presentada por Aubertin ${ }^{12}$, y el texto es citado por Messerli (1989: 26):

Auburtin había ya hecho esta distinción entre habla y lenguaje en su intervención en la Sociedad de Antropología: la facultad que él cree poder localizar en los lóbulos anteriores no es el lenguaje en tanto que 'creación de signos determinados que representan ideas determinadas' como lo había definido Gratiolet, sino más bien 'la facultad de coordinar los movimientos propios del lenguaje. Los enfermos de los cuales he hablado (...) no han olvidado el sentido de las palabras puesto que pueden responder por escrito' (1861: 278).

A lo que se agregan las explicaciones de Messerli, a partir de los intentos de Trousseau y Broca por aclarar el concepto de lenguaje y el concepto de afasia (Messerli 1989: 26):

(...) Estamos en el derecho de concluir que lo que se ha descrito bajo el nombre de afemia no es una afasia sino lo que, 45 años más tarde, Pierre Marie llamará una anartria. Por razones etimológicas, Trousseau había preferido el nombre de afemia y es el suyo el que prevaleció. Desgraciadamente, la afasia de Trousseu no corresponde a lo que Broca había descrito con el término de afemia. Broca se dio cuenta de esta desviación y aclara las cosas en un artículo de 1869 (el último sobre el lenguaje) en el cual distingue entre los trastornos del habla: 1) La alogia (en el contexto de demencias), 2) la amnesia verbal (nuestra afasia); 3) la afemia (nuestra anartria); 4) la alalia mecánica (nuestras disartrias).

Es interesante destacar que, en estos textos de discusión terminológica, está asociado el término lenguaje con los de 'habla' 'lenguaje articulado', 'facultad para' 'abolición de' y 'lugar donde reside la facultad'.

Se alude también, en los textos, al concepto de lenguaje como punto de partida para acercarse a una serie de "trastornos" específicos de lenguaje que hacía falta nombrar y denominar, tales como afemia, afasia, anartria, amnesia verbal, y alogia.

Estas "alteraciones", relacionadas con "el lenguaje en general" y "con la abolición de la facultad específica para" eran presentadas por los pacientes y, por lo novedoso de los hallazgos, no habían recibido una denominación "consensual", "homogénea" ni "oficial".

Llama también la atención que "la facultad del lenguaje en general" así como "la facultad de coordinar los movimientos propios del lenguaje" y "la facultad de articular" pueda alterarse y, sobre todo, localizarse en un punto específico del cerebro: el lugar donde ocurría una lesión.

A partir de los textos mencionados, podemos señalar que el vacío encontrado en las fuentes consultadas, en lo referente a una posible definición de lenguaje por parte de la teoría localizacionista está vinculado, según nuestro criterio, con el objetivo de las teorías en estudio de nombrar y denominar una serie de patologías relacionadas con el lenguaje, y no en profundizar en lo que se etiquetaría con el término lenguaje. 
Sobresale de esta situación el hecho de que, independientemente de la poca delimitación de lenguaje con la que trabajó la línea localizacionista, esta poco definida facultad pudiera ubicarse, mediante su alteración, en lugares específicos del cerebro en los pacientes tratados en las investigaciones.

En otros textos más actuales, también se presenta un panorama similar, como en el siguiente texto de Nass, R. et al. (1998: 70), en el cual se discute la posibilidad de que una facultad lingüística esté localizada en el cerebro:

\begin{abstract}
Refiriéndose al caso de una niña de tres años, "a pesar de su edad, esta afasia de conducción y la localización de la lesión se asemeja a la de un adulto. Las dificultades relacionadas con la memoria multimodal parecen subrayar lo que ha sido mejor descrito como una afasia de conducción. (...) En 1998 en Academic Press la localización neuroanatómica de la tradicional afasia cortical y en un grado menor, pero no menos significativo, el grado de afasias subcorticales está generalmente bien estandarizado para el adulto (Heilman y Valenstein, 1993; Wallesch y Papagno, 1988). La frecuencia elevada de la afasia no fluida con escasa comprensión durante la infancia ha permitido sugerir que tal localización no está presente en la infancia, porque el lenguaje no está bien (típicamente) localizado en el hemisferio izquierdo hasta en la adolescencia" (Lenneberg, 1975; Brown y Hecaen, 1976).
\end{abstract}

También se observa la tendencia localizacionista en las siguientes citas del texto de Van Petten, C. (2006) en el cual, mediante la técnica de potenciales evocados (ERPs) se ha logrado medir la actividad eléctrica del cerebro para estudiar aspectos diversos del procesamiento del lenguaje:

Varios diversos componentes del ERP han sido útiles para entender diferentes aspectos del tratamiento del lenguaje. Dentro del amplio número de componentes del ERP, que han mostrado sensibilidad hacia el tratamiento del lenguaje, el N400 ha sido el más comúnmente usado hasta la fecha (Van Petten 2006: 279- 280).

Una de las ventajas de usar los ERPs en el estudio del tratamiento del lenguaje ha sido la exquisita resolución temporal de la actividad eléctrica del cerebro, la cual puede ser usada para evaluar la secuencia de las operaciones cognitivas que intervienen entre la llegada de una señal sensorial a la corteza y la comprensión de este significado, papel sintáctico en una oración particular o el programa motor requerido para nombrar este significado (véase Hahbe y Friederici, 1999; Van Petten, 1995; Van Petten, Coulson, Rubin, Plante, y Parks, 1999; van Turennout et al., 1998) (Van Petten 2006: 281).

Y agrega:

(...) Esos resultados sugieren que a pesar de que el lóbulo frontal es crítico para varios aspectos del tratamiento del lenguaje, sí hace una pequeña contribución directa al N400. (...) Un tercer estudio confirma que el daño en la corteza prefontal, puede dar lugar a un ERPs anormal durante la comprensión de lenguaje pero esas anormalidades están en componentes diferentes a los de N400 (Van Petten 2006: 284).

\title{
En otro apartado:
}

Damasio y colaboradores reportan un déficit específico en el acceso a palabras para referirse a fotos de personas y animales, a pesar de que el concepto se conozca (por ejemplo, un paciente que no es capaz de producir la palabra zorrillo, pero responde 'un animal común que anda por aquí, negro y blanco, huele mal, a menudo es visto aplastado en la carretera'). Estos autores sugieren que el lóbulo temporal no es un almacén del conocimiento conceptual, pero sí un intermediario crucial entre la forma de las palabras y la memoria semántica (Damasio, Grabowski, Granel, Hichwa, y Damasio, 1996) (Van Petten 2006: 85).

Como se deduce de las citas anteriores, la posición con respecto a la teoría localizacionista es bastante ambigua. Por una parte, el texto de Van Petten es del 2006, y en 
ningún momento del artículo se alude directamente a las teorías localizacionistas; sin embargo, en los numerosos ejemplos citados, hay constante alusión a los déficit, y a las substancias que contribuyen a ubicar tales déficit en el cerebro.

Además, el texto pareciera también trabajar en la línea global, por lo actual de la publicación, por la tecnología de alto nivel que emplea y la utilización del término "tratamiento de lenguaje", que podría implicar la búsqueda de procesos involucrados y de todos los recursos mentales para que estos ocurran.

Incluimos este material porque consideramos que, en él, la frontera entre lo que se ha etiquetado como localización y como global no está totalmente delimitada. Pensamos que lo anterior ocurre no solo por elección de los investigadores, sino como resultado de la historia de los estudios sobre el lenguaje y el cerebro.

En lo que se refiere al lenguaje, el texto, igual que los presentados al inicio, menciona frecuentemente el término, pero no lo define ni lo ubica dentro de ninguna tendencia. Lo que podríamos decir es que, en textos de este tipo, coinciden los términos lenguaje (en general) y los términos pronunciación, forma de palabras y semántica (en particular) de manera aislada así como acompañados de una clasificación "patológica".

\subsection{Definiciones relacionadas con el núcleo 2}

El núcleo 2 se refiere al presupuesto de que "el procesamiento del lenguaje se ubica en uno de los hemisferios, el izquierdo (lateralización del lenguaje)".

Al respecto, los siguientes datos mencionados por De la Fuente et al. (1999: 41):

Un paso adelante en el campo de las localizaciones cerebrales le valió a Roger Sperry del Instituto tecnológico de California, el Premio Nobel de Fisiología y Medicina de 1981. Sperry (27) estudió sujetos a quienes se les había seccionado la comisura interhemisférica como último recurso para controlarles crisis convulsivas que habían sido incoercibles. Lo que estos estudios revelaron fue que cada uno de los hemisferios "siente", "percibe", "memoriza" y "conceptualiza" en forma independiente; que los hemisferios izquierdo y derecho están especializados en el procesamiento de diferentes clases de información. El izquierdo es más eficaz en el lenguaje, la escritura y el cálculo, y el derecho en otras tareas especiales complejas. Algunos trabajos sugieren que la "intuición" es una función del cerebro derecho, en tanto que el análisis lo es del izquierdo. Según Sperry hay dos corrientes de advertencia consciente; dos mentes separadas que en condiciones normales, cuando las conexiones interhemisféricas están intactas, funcionan dándonos una experiencia consciente unificada.

También el texto de Damasio (2001: 101) se refiere a la lateralización del lenguaje:

El ejemplo de dominancia más conocido se relaciona con el lenguaje. En más de $95 \%$ de personas, incluidos numerosos zurdos, la función del lenguaje depende de estructuras situadas en el hemisferio izquierdo. Otro ejemplo de dominancia, esta vez relacionado con el hemisferio derecho, tiene que ver con la percepción de las informaciones sensoriales procedentes del cuerpo: la representación del estado funcional de las víceras, por una parte, y las del estado funcional de los músculos esqueléticos de los miembros, del tronco y de la cara, por otra parte, se combinan en una carta dinámica coordinada. Más exactamente, no hay combinación en una sola carta, sino más bien interacción y coordinación entre señales que llegan al nivel de varias cartas distintas. En este cuadro, los mensajes relativos a los lados izquierdo y derecho del cuerpo encuentran su máximo de convergencia en el hemisferio derecho, en el nivel de las tres regiones del córtex somatosensorial mencionado arriba. De manera curiosa, la representación del espacio fuera del cuerpo, así como los procesos emocionales, son objeto de una dominancia hemisférica derecha. Eso no quiere decir que el cuerpo o el espacio no estén representados en las estructuras equivalentes del hemisferio izquierdo. Simplemente, las representaciones son diferentes: a la izquierda, son probablemente parciales y no son objeto de una integración funcional. 
En los textos anteriores, se ilustra, mediante ejemplos relacionados con el lenguaje, otro de los presupuestos de la teoría localizacionista: el lenguaje está ubicado en el hemisferio izquierdo del cerebro, en oposición a otras funciones que estarían lateralizadas en el hemisferio derecho.

En estos textos, de nuevo, se menciona el término lenguaje aunque este no se defina. Aparecen elementos específicos que acompañan o sustituyen al término lenguaje en diferentes momentos, tales como: escritura y tareas especiales complejas, por ejemplo.

También se refieren a un lenguaje (en general y sin definir) que está localizado en el cerebro humano. Además, interesa resaltar, en los textos, que ya que ese lenguaje está localizado en el cerebro, es necesario precisar cuál de los dos hemisferios está mayormente encargado de realizar las funciones relativas al lenguaje.

La línea de la lateralización del lenguaje ha sido muy productiva hasta la fecha. Independientemente de la concepción de lenguaje que se maneje, y de la teoría localizacionista o global que se siga, hay un hecho contundente que sigue generando investigaciones relacionadas con la lateralización: el que, en los pacientes afásicos, sea posible encontrar, a partir de las técnicas modernas, una lesión ubicada en el hemisferio izquierdo, en el derecho, o en ambos.

\section{3. Definiciones relacionadas con el núcleo 3}

El núcleo 3 plantea la existencia de una conexión paralela entre cada tarea lingüística y cambios químicos,celulares y configuracionales en áreas cerebrales particulares. Además, señala que las funciones psicolingüísticas se pueden aislar.

Un primer dato lo presenta Crystal (1993: 162), al tratar de aclarar cómo se entiende "habla" y "trastornos de lenguaje" cuando se estudian los daños de las funciones motoras de los órganos vocales:

\footnotetext{
Bajo el encabezamiento de trastornos del habla, en su más amplio sentido, por tanto, se sitúa todo problema que surge de un daño de las funciones motoras de los órganos vocales -trastornos en la anatomía, fisiología o neurología de los sistemas relacionados. En esta acepción general, los trastornos de la producción de voz, fluidez y articulación podrían resumirse como "trastornos de habla", dado que en ningún caso está afectada la formulación del significado, sino solo su transmisión a través del sonido. Bajo la denominación de trastornos del lenguaje se agrupan aquellos problemas que afectan a la formulación y comprensión del lenguaje -en concreto la afasia- y también un amplio espectro de trastornos de desarrollo y psicológicos. En esta distinción existen varias cosas equivocadas. Primero, se centra exclusivamente al sonido como medio de comunicación, como opuesto al visual o táctil -énfasis comprensible, pero teóricamente limitado-. Segundo, concede prioridad a los trastornos motores, como opuestos a los sensoriales. ¿Por qué no se menciona la audición, en el sentido anterior, dado que se trata de una categoría de problema comparable en líneas generales a las del habla? En tercer lugar, existe una confusión debida al significado cotidiano del término "habla" para designar al "lenguaje hablado" -en el cual, inevitablemente, interviene el significado.
}

El texto anterior presenta varios elementos interesantes relacionados con la definición de trastornos, de habla, y otros. Sin embargo, en este apartado nos referiremos únicamente a la relación entre el término lenguaje con un postulado de la teoría localizacionista: la posibilidad de aislar las tareas psicolingüísticas y, sobre todo, la conexión entre cada una de esas tareas psicolingüísticas y los cambios o daños en áreas cerebrales específicas.

En esta cita hay una referencia clara a tareas psicolingüísticas específicas: hablar, escuchar, emitir un sonido así como a procesos psicolingüísticos más generales como formular un significado, formular y comprender lenguaje. 
El texto discute las denominaciones de los trastornos de lenguaje y la exclusión e inclusión de algunas tareas lingüísticas dentro de esas categorías, pero no hay discusión en lo relacionado con el vínculo entre una tarea lingüística y un daño en un área cerebral determinada.

También el siguiente texto presenta elementos que coinciden con la tendencia localizacionista: la referencia a una lesión localizada en un hemisferio, cerca de un área cerebral específica, y sus consecuencias en la producción lingüística. El texto es parte de un resumen de la investigación de Tabor et al. (2006: 171):

\footnotetext{
El cerebelo posterolateral derecho participa con el lóbulo frontal izquierdo en la selección y producción de palabras. Con la ayuda de la técnica fMRI, examinamos si la actividad cerebelar conecta los hemisferios en paralelo con el reclutamiento de una compensación putativa en la región homóloga frontal derecha en una lesión afásica.

(...) Los pacientes afásicos que participaron en la investigación tenían lesiones debidas a un daño en el hemisferio izquierdo, cerca de la zona de Broca.

(...) Se encontró que la parte frontal derecha y los circuitos cerebelares izquierdos parecen ser relevantes para recuperar los residuos de las funciones verbales.
}

\section{Definiciones de lenguaje relacionadas con la teoría global}

Al inicio de esta investigación, tal como se pensaba en el caso de la definición de lenguaje relacionada con la teoría localizacionista, se intentó también buscar información sobre el concepto de lenguaje que dirigía los estudios de corte holístico. Sin embargo, se encontró, también en el caso de la teoría global, un vacío en lo referente al concepto de lenguaje. Igual que como sucedió con el material consultado sobre localización, en las fuentes sobre la teoría global, la aparición del término lenguaje es numerosa pero no precisa.

Por lo tanto, procedemos, tal como se hizo en los apartados sobre localización, a plantear los mismos tres grandes núcleos; pero, esta vez, desde la perspectiva holista, es decir, construidos a partir de la oposición y refutación de los planteamientos localizacionistas.

Con respecto a los núcleos escogidos en este trabajo, la teoría global los plantea de la siguiente manera:

a) La teoría global se opone a la idea de que el lenguaje esté depositado materialmente en el cerebro, y mucho menos en ciertas zonas de la masa cerebral. También refuta la argumentación de que solo algunas áreas del cerebro, como el área de Broca, estén implicadas en la actividad lingüística.

b) La teoría global estudia la relación entre los dos hemisferios cerebrales y también recoge evidencias de los pacientes con daños cerebrales en uno u otro hemisferio. Sin embargo, en el momento de realizar los análisis de los casos, la teoría global estudia el caso particular y observa todos los elementos, procedentes de los dos hemisferios, que puedan intervenir en la actividad lingüística perturbada o preservada.

c) La tendencia holista refuta la posibilidad de una conexión paralela entre cada tarea lingüística y cambios celulares y configuracionales en áreas cerebrales particulares. También niegan que se puedan aislar las funciones psicolingüísticas. 


\title{
5.1. Definiciones de lenguaje relacionadas con el núcleo 1
}

El núcleo 1 presenta la siguiente posición de la teoría holista:

"La teoría global se opone a la idea de que el lenguaje esté depositado materialmente en el cerebro, y mucho menos en ciertas zonas de la masa cerebral. También refuta la argumentación de que sólo algunas áreas del cerebro, como el área de Broca, estén implicadas en la actividad lingüística".

En las citas que se presentan a continuación se observa la posición de los seguidores de la teoría global en relación con un concepto y ubicación del lenguaje.

Caplan (1987: 166-167) señala claramente la oposición entre la tendencia localizacionista y global:

\begin{abstract}
Durante muchos años se ha pensado que el tema principal de las teorías neurolingüísticas era la discrepancia sobre las relaciones lenguaje-cerebro entre los "localizacionistas" y los "holistas". Se decía que los localizacionistas creían, más o menos, que la manera como el cerebro procesa el lenguaje es por medio del funcionamiento de centros y conexiones. El conexionismo es el arquetipo del localizacionismo. Por el contrario, los holistas sostenían que todo el cerebro (o, al menos, grandes zonas del cerebro) se encarga de las tareas individuales del funcionamiento del lenguaje.
\end{abstract}

Lo anterior se complementa con lo expuesto por Crystal (1993: 121):

Desde los primeros años, no obstante, apareció evidencia contraria a esta forma (la localización) estricta de hipótesis (entre los principales oponentes se encontraban Hughlings Jackson y Henry Head). Con un área de Broca sin dañar, por ejemplo, un paciente podía manifestar un habla desordenada; y al revés, la lesión del área de Broca no era inevitablemente y en el mismo grado, causa de problemas de habla. Como dijo Jackson: "localizar el daño que destruye el habla, y localizar el habla, son dos cosas diferentes.

Las citas anteriores son muy claras en la manera de oponer la tendencia localizacionista a la global. Insistimos en el hecho de que, tal como sucedió en los apartados dedicados a la teoría localizacionista, también en este caso no hay una intención de definir lenguaje. Más bien, se utiliza el término de manera general y lo que interesa es señalar como posible o no posible el que todo lo etiquetado bajo el término lenguaje pueda estar localizado en áreas específicas del cerebro.

\subsection{Definiciones de lenguaje relacionadas con el núcleo 2}

El núcleo número 2 debate el tema de la especialización hemisférica. A saber:

La teoría global estudia la relación entre los dos hemisferios cerebrales. Recoge evidencias de pacientes con daños cerebrales en uno u otro hemisferio para observar su situación. En el momento de realizar los análisis de los casos, la teoría global estudia casos particulares y observa en ellos todas las evidencias, procedentes de los dos hemisferios, que puedan intervenir en la actividad lingüística perturbada o preservada.

La siguiente cita, de Aram, 1992, encontrada en Starck y McGregor (1997: 222) presenta un enfoque global:

Hasta hace poco era ampliamente aceptado que el desarrollo de los desórdenes de lenguaje estaban relacionados con el malfuncionamiento del hemisferio cerebral izquierdo, dominante en el lenguaje. Sin embargo, los niños con desórdenes en el desarrollo del lenguaje presentan más deterioro en todos los dominios del lenguaje que los niños que, temprano en la vida, presentan lesiones localizadas en el hemisferio izquierdo. Esto hace suponer que, en los niños con lesiones izquierdas, la plasticidad neuronal 
permite a las partes no dañadas del hemisferio izquierdo realizar las funciones normalmente asumidas por el hemisferio izquierdo (Aram 1992).

La cita anterior compara el deterioro del lenguaje en niños que han padecido una lesión cerebral izquierda y aquellos que tienen desórdenes del desarrollo del lenguaje. Lo importante de este texto para nuestro tema es el hecho de que, nuevamente, hay múltiples referencias al lenguaje, aunque éste no se defina. También destacamos el hecho de que se cuestione, de acuerdo con la teoría global, el predominio del hemisferio izquierdo sobre el derecho en las actividades lingüísticas.

La plasticidad neuronal ha sido uno de los elementos considerados para debatir la lateralización del lenguaje. Como lo señala el texto antes citado, la plasticidad neuronal, observable con más fuerza en los niños, es un elemento que permite pensar en que los dos hemisferios cerebrales (y no solo el izquierdo) son importantes en la realización de tareas lingüísticas.

En la misma línea antes expuesta, la investigación de Frishkoff, G. (2006: 2), en la que se cuestiona los papeles diferentes que se le han asignado a los dos hemisferios cerebrales:

(...) ¿Hay diferencias en cómo el hemisferio derecho y el izquierdo procesan diferentes patrones de actividad lingüística relacionadas con el procesamiento de información semántica?

(...) Aquí, nos referiremos a la Hipótesis de Equivalencia, la cual se refiere a que asociaciones (semánticas) fuertes y débiles deberían ser procesadas de manera similar por los dos hemisferios.

Otra cita influenciada por la teoría global es la de Starck, R. y K. McGregor (1997: 222):

Estos hallazgos han permitido a algunos investigadores proponer que los desórdenes del lenguaje deben estar relacionados con una influencia mayor del cerebro más que con anomalías localizadas únicamente en el hemisferio izquierdo (Aram \& Eisele, 1994; Plante, Swisher, Vance, \& Rapcsak, 1991).

Y de los mismos investigadores (1997: 223):

El propósito del primer estudio es comparar a dos niños con lesiones cerebrales en distintos hemisferios, con respecto al desarrollo de su recuperación del lenguaje. Se predijo que esta recuperación sería más rápida para Ruth, la niña lesionada en el lado derecho, que para Ell, la niña lesionada del lado izquierdo.

Además, Starck y McGregor (1997: 242):

Aram and Eisele (1994) señalaron que el daño cerebral limitado a ciertas regiones específicas del hemisferio izquierdo no es suficiente para producir un déficit a largo plazo en los niños. Estos autores sugirieron que los modelos que involucran áreas difusas del cerebro podrían ser más adecuadas como una explicación de los daños específicos de lenguaje.

(...) nuestros datos proveen fuerte evidencia para la dominancia del hemisferio izquierdo en la adquisición de lenguaje también. Ruth, la niña con lesión derecha, mostró pocas características de lenguaje y de daño conceptual Así, el daño cortical difuso de manera aislada no puede ser la causa del déficit lingüístico. Alguna influencia del hemisferio izquierdo, tanto unilateral izquierdo como influencia bilateral, puede ser necesario.

Finalmente, con los avances de la tecnología, la conclusión de que el daño cerebral resulte de la influencia subcortical difusa o aislada deber ser evaluada para obtener datos más decisivos. Esperamos que nuestra comparación entre los niños en estudio provea indicios de aspectos conductuales para futuros estudios que exploren las bases neurológicas de los deterioros en el desarrollo del lenguaje.

En las citas expuestas, hallamos elementos que señalan la influencia de la teoría global en la manera de considerar la intervención de los dos hemisferios cerebrales en la planificación y ejecución de las tareas lingüísticas.

En los textos se debate el papel protagónico que ha tenido el hemisferio izquierdo en las actividades lingüísticas y se recomienda mayor investigación en esa línea. Como observamos, 
los textos antes citados mencionan varias veces el término lenguaje. Y, de nuevo, no lo definen ni lo contextualizan. En su lugar, el concepto es una categoría en la cual se incluyen los diferentes procesos relacionados con las actividades lingüísticas que se han deteriorado.

Finalmente, los textos coinciden con las propuestas de la teoría global, en la medida en que cuestionan el predominio del hemisferio izquierdo en las actividades relacionadas con el lenguaje. Como dijimos antes, dentro de la línea global, no se descarta la influencia del hemisferio izquierdo, pero se toma en consideración la influencia de los dos hemisferios en la planificación y ejecución de actividades lingüísticas.

\subsection{Definiciones de lenguaje relacionadas con el núcleo 3}

El núcleo 3 se refiere a la relación entre las tareas lingüísticas y su conexión con cambios configuracionales en áreas cerebrales específicas. A saber:

La tendencia holista refuta la posibilidad de una conexión paralela entre cada tarea lingüística y cambios celulares y configuracionales en áreas cerebrales particulares. También niegan que se puedan aislar las funciones psicolingüísticas.

Al respecto, las siguientes citas aportan datos que cuestionan, a partir de datos empíricos, la posibilidad de relacionar una tarea lingüística (en este caso la tarea de la significación), con los cambios químicos y configuracionales en áreas cerebrales específicas.

A continuación, los textos de Frishkoff (2006: 3):

\footnotetext{
La hipótesis de que las asociaciones (semánticas) fuertes y débiles podrían evocar diferencias cualitativas en la activación cerebral descansan en la idea de que los diferentes procesos cognitivos soportan la activación de relaciones semánticas cerradas (o fuerte, focal) versus remotas (o amplia, difusa). (...) En su formulación original, del modelo de activación de extensiones, Collins y Loftus (1975) sugirieron que los conceptos relacionados "están almacenados juntos" en un espacio psicológico y neuronal.
}

Como se observa, el texto anterior cuestiona la propuesta del modelo de Collins y Loftus (1975) en lo relacionado con la posibilidad de localizar en un espacio particular los conceptos relacionados semánticamente. Se duda de que para las tareas lingüísticas relacionadas con la significación exista un espacio cerebral específico, y cambios neurales que den cuenta de tal localización. Sobre el concepto de lenguaje, podemos decir que no se usa en su acepción general. Más bien, se utiliza en este texto el término específico de "relación semántica". Independientemente del término utilizado, lenguaje o relación semántica, la terminología se da por sabida. Interesa destacar la posibilidad o imposibilidad de que la etiqueta escogida (lenguaje o tarea específica) pueda aislarse y localizarse específicamente en un área cerebral particular.

El mismo investigador, G. Frishkoff (2006: 6), cuestiona la hipótesis sobre el tratamiento semántico para resaltar la participación de los dos hemisferios en el tratamiento semántico y, por ende, la imposibilidad de localizar en un área específica la tarea de significación semántica:

El patrón de asimetrías observado aquí no apoya la versión simple de la Hipótesis de Complementariedad, la cual postula que el hemisferio izquierdo está especializado en el tratamiento de asociaciones cerradas (...) mientras que el hemisferio derecho se encarga de un rango amplio de relaciones semánticas (Burgess \& Simpson, 1988; Chiarello, Burgess, Richards, \& Pollock, 1990). 
Presentamos a continuación textos que se refieren a la imposibilidad de aislar las funciones psicolingüísticas (en este caso las relacionadas con la pragmática) con la pretensión de encontrar áreas cerebrales específicas que se activen o se modifiquen, celular o químicamente, cada vez que se ejecuten o se planifiquen tales tareas.

Al respecto, Kasher, Batori et al. (1999: 567) parten de un presupuesto, la especialidad hemisférica:

Se dice a menudo que los pacientes con lesión cerebral derecha (RBD) muestran deterioros selectivos en el lenguaje natural pragmático, por ejemplo, el uso del lenguaje en contexto. Se ha presupuesto que el hemisferio derecho $(\mathrm{RH})$ involucrado en la pragmática incluye el tratamiento de la prosodia, las emociones y la comunicación no verbal, ciertos aspectos de los actos de habla, especialmente los estilos indirectos, y el lenguaje figurativo, incluyendo las frases idiomáticas y las metáforas así como el humor, las inferencias y el discurso (Tompkins, 1995). Todas esas funciones se muestran en la Batería de Comunicación del Hemisferio Derecho (RHCB) de Gardner y Brownell (1986).

Y lo refutan (Kasher, Batori et al. 1999: 569 y 590):

\begin{abstract}
El resultado más importante de este estudio (el que ellos realizan) es que ambos hemisferios cerebrales contribuyen en el mismo grado en el procesamiento de implicaturas. La lesión de cualesquiera de los hemisferios altera la producción en nuestra Batería de Implicaturas (IB).

No hay evidencia de disociación hemisférica en diferentes implicaturas, pero el resultado negativo debe interpretarse con precaución.

En el H.I, las implicaturas verbales y no verbales están altamente correlacionadas. Contrariamente, en el HD, hubo fuertes correlaciones entre las implicaturas verbales y hubo fuertes correlaciones entre las implicaturas no verbales, pero no hubo correlaciones entre las implicaturas verbales y no verbales. Concluimos que solamente el hemisferio izquierdo incluye un procesador de implicaturas.

Como conclusión, ambos hemisferios parecen estar involucrados en el tratamiento pragmático de las implicaturas, pero el responsable de los mecanismos cognitivos parece ser diferente en cada uno de los hemisferios, Las implicaturas verbales y no verbales no están selectivamente lateralizadas en el H.I. y en el H.D., respectivamente. El H.D. parece incorporar un mosaico de procesadores específicos, mientras que el H.I. activa un procesador más general, el cual, sin embargo, no es extensible a todas las funciones del lenguaje (...). Por otra parte, el mosaico del H.D. parece realizar más funciones no lingüísticas que las que realiza el procesador general del H.I. Ningún lado muestra una fuerte localización anatómica de las implicaturas.
\end{abstract}

Los textos de Kasher et al. (1999), como ya lo señalamos, presentan evidencias para oponerse a la idea de una posible localización cerebral de las tareas lingüísticas. Como se lee en las citas antes expuestas, su investigación es sobre las tareas pragmáticas y su posible localización en un hemisferio específico. Nos interesa resaltar de este trabajo el hecho de que cuestione el predominio de un hemisferio sobre otro y la posibilidad de aislar las tareas lingüísticas (pragmáticas en este caso) para ubicarlas en un área específica del cerebro. Como vemos, la investigación de estos autores es acorde con la teoría global.

Y finalmente, un texto tomado de la Revista de Alteraciones de la Comunicación en el cual, de manera contundente, se niega la hipótesis localizacionista de las tareas lingüísticas (1994: 305):

Recientes estudios anatómicos sugieren que las funciones cerebrales no tienen lugares anatómicos precisos (Andersonn et al. 1990). Los estudios que utilizan la técnica de estimulación cortical directa han sido más dramáticos al respecto, y han demostrado que no se puede predecir la ubicación de la región perisilviania capaz de alterar la denominación confrontacional en múltiples pacientes. 


\section{Conclusiones}

En los textos presentados, consideramos haber expuesto la influencia de la teoría localizacionista y global sobre la manera en la que se concibe el lenguaje (así como sus características) en las investigaciones de orientación psicolingüística.

Tanto en los textos influenciados por la teoría localizacionista como con la global, hubo un mismo patrón: la ausencia de una definición de lenguaje. En su lugar, el término lenguaje alterna con sus especificaciones : semántica, pragmática y otras.

Interpretamos la ausencia de una definición precisa de lenguaje como evidencia de la poca o nula definición que reciben los "presupuestos" en las prácticas de las disciplinas que trabajan con el lenguaje.

En este caso, la definición de lenguaje toma la forma general de los presupuestos: la invisibilidad.

Sin distinción de año de publicación ni de grado de modernidad de las técnicas empleadas, en las investigaciones coexisten rasgos postulados por las teorías holistas y por las globales.

Las posiciones actuales ya no son solo localizacionistas ni solo globales. En su lugar, se acepta que los correlatos entre lenguaje y cerebro tienen una base anatómica, aunque su estudio no se limite a lo anatómico.

Actualmente se toman en cuenta, en los estudios sobre el procesamiento del lenguaje, la influencia de factores variados como la cultura y las circunstancias individuales.

El reto consiste en investigar las habilidades lingüísticas desde un marco funcional general, sin negar que éstas tengan su depósito y desarrollo en el cerebro.

Lo anterior es importante porque, tal como lo presentaron los textos citados, aunque en algunos aspectos las teorías en estudio son totalmente opuestas, se acercan en varios aspectos.

Ambas teorías coinciden en la naturaleza y localización de síntomas. Las dos teorías interpretan los síntomas como indicadores de un déficit y les atribuyen un papel en las funciones lingüísticas. La diferencia entre ellas radica en la manera en que éstas interpretan tales deterioros.

En las teorías localizacionistas la noción de síntoma hace referencia a "alteraciones, retrasos, falta de, deterioro en" y ese síntoma se asocia con un lugar en el cerebro. Las teorías globales también interpretan el síntoma como "alteraciones"y "déficit", pero no lo ubican en un lugar específico del cerebro; más bien, lo contextualizan para señalar en qué medida la actividad cerebral, en su conjunto, coexiste con el síntoma y las consecuencias que esto tiene en cada paciente estudiado. Se interpreta el síntoma a partir de su relación con los daños anatómicos o alteraciones funcionales ocurridos en otras áreas o zonas diferentes de la señalada como "lugar o zona perturbada".

De esta manera, las teorías en estudio discrepan ampliamente en la naturaleza y localización de las funciones del lenguaje. La teoría localizacionista propone ubicar las funciones del lenguaje en áreas específicas del cerebro, mientras que las teorías globales proponen la búsqueda de conexiones paralelas básicas a pesar de que la diversidad funcional no siempre responda a correlatos materiales prefijados. Estos planteamientos buscan considerar componentes funcionales (psicológicos o lingüísticos) que no se ligan directamente con un centro o con un proceso cerebral. 


\section{Notas}

1. Los neurólogos Paul Broca en Francia y Carl Wernicke en Alemania llamaron la atención del sector médico por sus estudios sobre pacientes que habían sufrido una lesión cerebral. De manera independiente, Broca y Wernicke habían adelantado que la destrucción de regiones específicas del cerebro originaban un problema particular de lenguaje en sus pacientes. El problema en cuestión se denominó en términos técnicos, afasia. Tomado de Damasio, Antonio. 2001. L'erreur de Descartes. Paris: Editions Odile Jacob, 43.

2. El enfoque localizacionista buscaba centros o áreas específicas del cerebro como responsables de las diferentes funciones mentales. Tomado de Ortiz, T. 2002. Historia de la neuropsicología del lenguaje. Madrid. CEPE, S.A, 38.

3. La línea global u holística fue encabezada por Flourens (1794-1867), como respuesta a la tendencia localizacionista. Flourens afirmó que el cerebro funciona de acuerdo con una equipotencialidad o equivalencia de todas sus partes, en forma de un todo funcional y actuando, en una palabra, de forma holística. Para este investigador, los lóbulos cerebrales participan en el conjunto de las funciones; tanto así, que una lesión de una parte del cerebro no anula una función, sino que la debilita. Así pues, la inteligencia o las diferentes funciones mentales superiores del hombre no dependerían de áreas específicas del cerebro, sino de la función global y holística del mismo.

4. Belinchón, M. 1992. Psicología del lenguaje. Investigación y teoría. Madrid: Editorial Trotta, S.A., 1743, Capítulo 1: "El concepto de lenguaje".

5. Belinchón, M. 1992. Psicología del lenguaje. Investigación y teoría. Madrid: Editorial Trotta, S.A.,19.

6. $\quad$ Op. cit:: 19-20.

7. Op. cit.: 20 .

8. Tomado de Belinchón et al., 30- 32.

9. Belinchón, 59- 64 .

10. Estos núcleos, como los hemos llamado, se han establecido a partir de la lectura de textos como Ramón de la Fuente. 1999. Biología de la mente. México: Fondo de Cultura Económica.

Springer, S. y G. Deutsch. 1994. Cerebro izquierdo, cerebro derecho. Barcelona: Editorial Gedisa, S.A., del capítulo "El lenguaje y su naturaleza psicológica" en Fernández, M. 1999. Introducción a la linguiística. Barcelona: Editorial Ariel, S.A. Así como en otros relacionados con el tema.

11. En 1861, P. Broca presentó ante la Sociedad Antropológica de París el caso de un enfermo, Monsieur Levergne, cuya autopsia había practicado en días anteriores. El paciente conservaba sus facultades intelectuales intactas pero había perdido el lenguaje y solo podía pronunciar una simple palabra: "Tan". Referencia tomada del capítulo "La localización de las funciones mentales en el cerebro", en Ramón de la Fuente.1999. Biología de la mente. México: Fondo de Cultura Económica, 32.

12. Aubertin es uno de los seguidores de las teorías localizacionistas. En 1861 publica 'Discusión sobre la forma y el volumen del cerebro' en Bulletin de la Société d'Anthropologie de Paris, 2, 209-220. 


\section{Bibliografía}

Aguado, Gerardo. 1999. Trastorno específico del lenguaje. Retraso del lenguaje y disfasia. Málaga: Ediciones Aljibe.

Belinchón, M. et al. 1992. Psicología del lenguaje. Investigación y teoría. Madrid: Editorial Trotta, S.A.

Caplan, David. 2006. "Effects of syntactic features on sentence picture matching in Broca's aphasics: A reply to Drai and Grodzinsky (2005)". Brain and Language. 96: 129- 139.

Crystal, David. 1993. Patología del lenguaje. Salamanca: Ediciones Cátedra.

Damasio A. 2001. L'érreur de Descartes. Paris: Editions Odile Jacob.

De la Fuente, Ramón y Francisco Álvarez Leefmans. 1999. Biología de la mente. México: Fondo de Cultura Económica.

Portier, Jean-Francois. 2001. Le langage. Nature, histoire et usage. Paris: Sciences Humaines Editions.

Eustache, F. y B. Lechevalier. 1989. Langage et aphasie. Séminaire Jean-Louis Signoret. Bruxelles. De Boeck-Wesmael, S.A.

Fernández P., Milagros. 1999. Introducción a la lingüística. Barcelona: Editorial Ariel S.A.

Frishkoff, G. (accepted 24 June 2006). Hemispheric differences in strong versus weak semantic priming: Evidence from event-related brain potentials. Learning Research and Development Center, University of Pittsburg, Pittsburg, PA 15260, USA.

Kasher, A, G. Batori et al. 1999. "Effects on Right and left Hemisphere Damage on Understanding Conversational Implicatures". Brain and Language. 68: 566- 590.

Messerli, P. 1989. “Une approche historique de l'aphasie”. En: Eustache, F. et Lechevalier, B. Langage et aphasie. Séminaire Jean Louis Signoret. Bruxelles: De Boeck Université.

Ortiz, T. 2002. Historia de la neuropsicología del lenguaje. Madrid: CEPE, S.A.

Nass, Ruth, Fern Leventhal et al. 1998. "Conduction Aphasia in a 3 Year-Old with a Left Posterior Cortical/Subcortical Abscess”. Brain and Language. 62: 70- 88.

Springer, S. y Deutsch. 1994. Cerebro izquierdo, cerebro derecho. Barcelona: Editorial Gedisa, S.A. 
Starck, Rachel E. y Karla McGregor. 1997. "Follow-up Study of a Right and a LeftHemispherectomized Child: Implications for Localization and Impairment of Language in Children". Brain and Language. 60: 222- 242.

Van Petten, C. et al. 2006. "Neural Localization of Semantic Context Effects in Electromagnetic and Hemodynamic Studies". Brain and Language. 97: 279- 293.

Virno, P. 2004. Palabras con palabras. Poderes y límites del lenguaje. Buenos Aires: Editorial Paidós. 